\title{
Succession of Resonances to Achieve Internal Wave Turbulence
}

\author{
Géraldine Davis, ${ }^{1}$ Timothée Jamin $\odot,{ }^{1}$ Julie Deleuze, ${ }^{1}$ Sylvain Joubaud $\odot,{ }^{1,2}$ and Thierry Dauxois $\oplus^{1}$ \\ ${ }^{1}$ Univ Lyon, ENS de Lyon, Univ Claude Bernard, CNRS, Laboratoire de Physique, 69367 Lyon Cedex 07, France \\ ${ }^{2}$ Institut Universitaire de France (IUF), 1 rue Descartes 75005 Paris, France
}

(Received 4 December 2019; revised manuscript received 30 March 2020; accepted 23 April 2020; published 21 May 2020)

\begin{abstract}
We study experimentally the interaction of nonlinear internal waves in a stratified fluid confined in a trapezoidal tank. The setup has been designed to produce internal wave turbulence from monochromatic and polychromatic forcing through three processes. The first is a linear transfer in wavelength obtained by wave reflection on inclined slopes, leading to an internal wave attractor which has a broad wave number spectrum. Second is the broadbanded time-frequency spectrum of the trapezoidal geometry, as shown by the impulse response of the system. The third one is a nonlinear transfer in frequencies and wave vectors via triadic interactions, which results at large forcing amplitudes in a power law decay of the wave number power spectrum. This first experimental spectrum of internal wave turbulence displays a $k^{-3}$ behavior.
\end{abstract}

DOI: 10.1103/PhysRevLett.124.204502

Internal gravity waves propagate within density stratified fluids moving under the influence of buoyancy forces [1]. Recently, they have been actively studied in particular because of their importance to mixing and transport in the ocean. For example, a coordinated observational campaign has been performed in the South China Sea [2], which is well known to contain breaking internal waves with amplitudes up to $200 \mathrm{~m}$. Their generation through the interaction between tides and bathymetry [3], their propagation and instability [4], and their interaction with oceanic currents are just some of the outstanding dynamics being observed. In particular, the study of mixing by breaking internal waves is relevant for the understanding of biological processes such as the vertical redistribution of zooplankton from the deep ocean [5] and the regeneration of the coral reef of Dongsha Atoll [6].

In the nonlinear regime, stratified fluid systems may develop turbulence simultaneously due to waves and vortices [7]. Describing the coexistence of each process is a challenge in itself. If stratified turbulence has been actively studied (see Ref. [8], and references therein), wave turbulence for internal waves is a relatively unexplored phenomenon. Wave turbulence describes physical systems with a large number of dispersive and nonlinear interacting waves [9] and has been applied to gravity [10], capillary [11] and inertial waves [12,13], as well as waves in magnetized fluids [14] and in elastic plates [15]. New applications have recently emerged in condensed matter (superfluid helium and Bose-Einstein condensates), in nonlinear optics [16], and, most recently, in the study of gravitational waves in the early Universe [17]. Internal waves are distinct from these waves [18], owing to their unusual dispersion relation. In this Letter, we present an experimental setup that allows us to observe efficient nonlinear energy transfers in frequency and wave number and so determine the signature of internal wave turbulence.
The experimental setup, sketched in Fig. 1(a), is a confined trapezoidal domain filled with a linear stratified fluid of density $\rho(z)$, in which $z$ is the vertical coordinate. Introducing the gravity $g$ and a reference density $\rho_{\text {ref }}$, the strength of the stratification is characterized by the buoyancy frequency $N=\sqrt{-\left(g / \rho_{\text {ref }}\right) \partial \rho / \partial z}$. In all the experiments, this is of the order $1 \mathrm{rad} / \mathrm{s}$. The energy is injected at large scale by means of a vertical boundary oscillating horizontally around its midhorizontal axis, with a half cosine shape $a(t) \cos (\pi z / H)$, in which $a(t)$ is the maximum horizontal displacement and $H$ is the depth. The volume is thus kept constant. The classical experiments are performed with a quasimonochromatic forcing, $a(t)=a_{0} \sin (\omega t)$. Here, we also examine impulsive and polychromatic forcings. The resulting velocity fields are
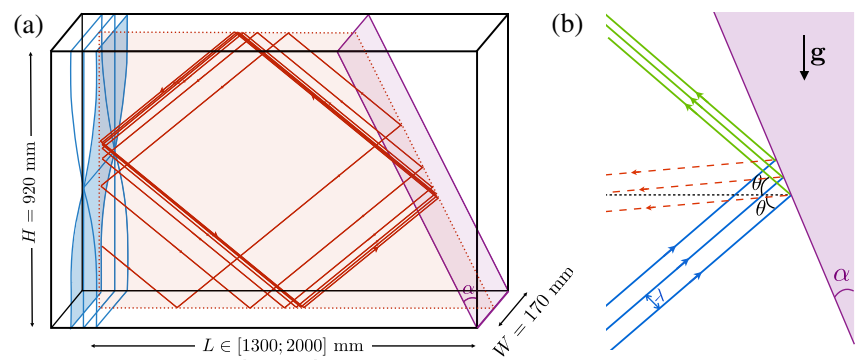

FIG. 1. (a) Experimental setup with the generator on the left of the tank and a slope inclined with an angle $\alpha$ on the right. An example of ray tracing from a single point located on the left-hand wall and corresponding to an oscillation at a given frequency $\omega= \pm N \sin \theta$ is shown in the case of a linearly stratified fluid with constant $N$ value. (b) Schematic reflection on a slope of an incident internal gravity beam (blue) with two wavelengths $\lambda$ in the transversal direction. The reflected beam does not follow the Snell-Descartes prediction (dashed red) but keeps the same angle with respect to the horizontal, or with the gravity $\mathbf{g}$, as shown by the green beam. 
measured using the classical particle image velocimetry method.

The dispersion relation of internal waves, $\omega=N \sin |\theta|$, is such that the frequency $\omega$ sets the angle $\theta$ of propagation of internal wave beams and also the ratio of the horizontal to vertical wave number. Reflections of internal waves on vertical or horizontal walls are analogous to optical reflection, preserving the angle with respect to the normal of the boundary. On the contrary, reflection on a slope interestingly leads to a ray with the same angle with the horizontal as the incident ray (in absolute value); this is a simple consequence of the preservation of the frequency $\omega$ after reflection with the dispersion relation. Figure 1(b) shows that this simple mechanism is even more interesting when considering a beam since both its width and its wavelength are reduced after reflection (notice that, for internal waves, the wave vector is orthogonal to the ray). As the group velocity is proportional to the wavelength, energy conservation leads to efficient energy focusing of the beam.

After multiple reflections, internal waves generated at a given frequency concentrate on a closed loop [19,20], as illustrated by a single ray traced in Fig. 1(a). The shape and rotational direction of the so-called internal wave attractor (IWA) are independent of the initial emitting point and thus of the spatial structure of the forcing. The IWA can be seen as a limit cycle, a prominent word in nonlinear physics, which arises from linear theory. Different angles of propagation set by different forcing frequencies lead to different attractors with simple or more complicated shapes: the dashed lines in Figs. 2(a)-2(f) present a few $(m, n)$ theoretical attractors, with $m$ reflections on the top and $n$ reflections on the slope.

Because of viscosity, IWAs have finite width as a consequence of the balance between focusing and dissipation as shown in Figs. 2(a)-2(f) [24,25]. The energy concentration, which is large just after focusing upon reflection from the slope, is progressively dissipated along the length of the attractor before being focused again by the slope. Recent experiments of IWA in two nontrivial threedimensional setups [26] have additionally shown that this is more than a beautiful mathematical curiosity.

It is important to emphasize that this energy focusing with corresponding transfer to small scales arises from linear theory. This is the first important ingredient in the context of wave turbulence as it increases the number of waves with different wave numbers in interaction.

Owing to energy focusing, the reflected beam has a larger amplitude and is therefore more prone to reach the threshold for triadic resonant instability (TRI) $[4,27,28]$ : a beam with primary frequency $\omega$ and wave vector $\mathbf{k}$ can excite from background noise two subharmonic waves with frequencies $\omega_{ \pm}$and wave vectors $\mathbf{k}_{ \pm}$, satisfying the temporal and spatial resonance conditions $\omega=\omega_{+}+\omega_{-}$ and $\mathbf{k}=\mathbf{k}_{+}+\mathbf{k}_{-}$. Any subharmonic may also become unstable through TRI and/or interact with another
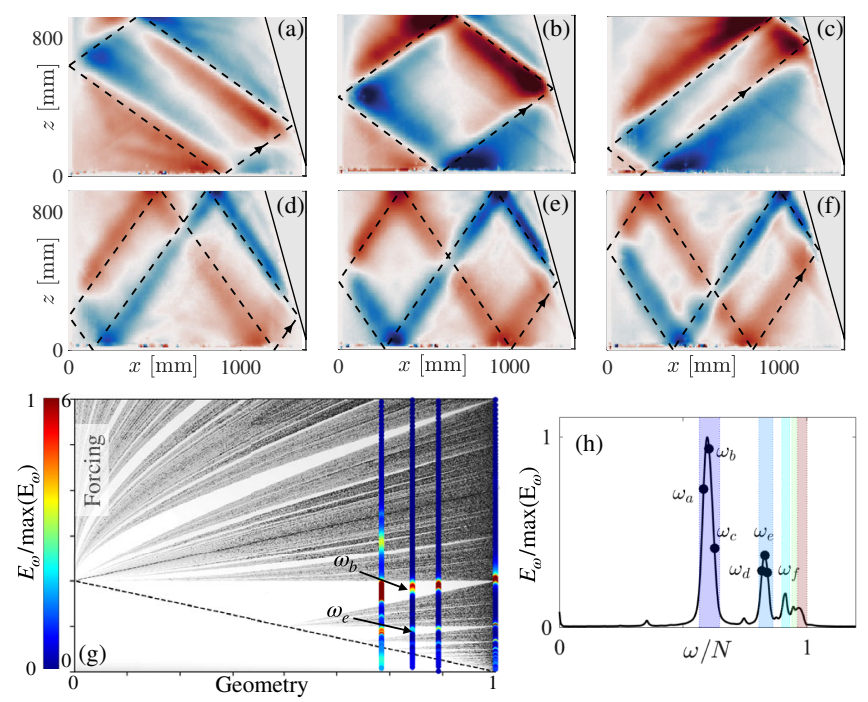

FIG. 2. (a)-(f) Spatial patterns obtained after an initial impulsive kick when the experimental signal is filtered around different frequencies shown with symbols in (h). One recovers $(1,1)$ attractors (a)-(c) and (2,1) attractors (d)-(f). The theoretically expected attractors for the geometry and corresponding frequencies are depicted with the dashed line. (g) In gray scale, Lyapunov exponents of the trapezoidal domain in the inviscid limit (from Ref. [21]) as a function of the geometry $[1-(2 H / L) \tan \alpha]$ and of the forcing $\left[(2 H / L) \sqrt{(N / \omega)^{2}-1}\right]$ which is decreasing while increasing the frequency. White regions correspond to strongly convergent attractors. (h) Energy spectrum measured experimentally via particle image velocimetry after an initial kick of the generator for $\alpha=15.5^{\circ}$. Vertical colored bands emphasize the theoretical frequency bands of internal wave attractors in the stationary regime. The same spectra for $\alpha=0^{\circ}, 11.1^{\circ}, 15.5^{\circ}$, and $21.4^{\circ}$ are superimposed on (f) as colored vertical lines, from right to left (corresponding graphs are presented in the Supplemental Material [22])

preexisting wave to generate a third one, the latter process being without any amplitude threshold. One thus obtains a necessary ingredient for wave turbulence: a physical mechanism providing multiple nonlinear interactions between waves of various wavelengths and frequencies.

Kicked attractor experiment.-Before building on this method to achieve internal wave turbulence, we consider the special case of transient impulsive forcing by analogy with musicians striking a tuning fork that resonates at a specific constant pitch.

If the internal wave generator is set into motion with time-dependent amplitude $a(t)$, which is nonzero in a short interval $\delta t \ll 2 \pi / N$, then a broad frequency spectrum will be simultaneously excited. We then consider the impulse response of the trapezoidal domain.

Additionally, plotting the Lyapunov exponent quantifies the exponential divergence of rays issued from close initial points. Figure 2(g) presents the theoretical prediction [21] in the inviscid limit. White tongues correspond to domains of existence of the different $(m, n)$ attractors. 
The impulse response of the different experiments is analyzed by considering the frequency content of the kinetic energy, $E_{\omega}(\omega)=\int\left[|\tilde{u}(x, z, \omega)|^{2}+|\tilde{w}(x, z, \omega)|^{2}\right] d x d z$, computed from the Fourier transform of the two components of the velocity field and integrated over the $(x, z)$ plane. Such energy spectra probe complete vertical lines in Fig. 2(g). In the absence of any inclined slope $(\alpha=0)$, when the geometry is 1 in Fig. 2(g), one gets a set of well-peaked discrete modes as shown by the rightmost vertical line (see the energy spectrum in the Supplemental Material [22]). This is expected because attractors, which require a sloping boundary, cannot exist in a rectangular domain. One thus recovers the discrete set of resonance frequencies of the rectangle. The width of these peaks observed experimentally is due to viscous effects. On the contrary, for nonzero $\alpha$, Fig. 2(g) shows that discrete modes are replaced by vertical bands located within the domain of existence of the different IWAs. These bands of peak energy are plotted in Fig. 2(h) for a given geometry. Filtering the velocity field at given frequencies allows us to disentangle the different responses to the initial impulsive kick. Figures 2(a)-2(f) present different attractors that appear when the signal is filtered around frequencies belonging to the $(1,1)$ and $(2,1)$ tongues of Fig. 2 (a). Note that only IWAs with short perimeters (i.e., with small $m$ and $n$ values) are visible in the presence of damping.

Introducing a slope therefore modifies the usual picture of a wave operator with discrete eigenmodes to some continuous spectrum, and understanding the linear response of these systems is a nontrivial question [29]. By taking advantage of such frequency band within one of the tongues we can efficiently force the system in a polychromatic way, thus increasing the number of waves in interaction. As almost all regions of the physical domain will be covered by an IWA, this characteristic also allows us to inject energy rather homogeneously. Both properties are beneficial in the study of wave turbulence.

Beyond the linear regime.-We first consider the transition to nonlinear dynamics resulting from monochromatic forcing $a(t)=a_{0} \sin \left(\omega_{0} t\right)$. Inspired by the transition to wave turbulence observed numerically in harmonically forced elastic plates [30], the amplitude of the generator $a_{0}$ is gradually (from 2 to $10 \mathrm{~mm}$ ) and slowly (over 750 oscillating periods) increased, as shown in Fig. 3(a). The frequency has been chosen within the $(1,1)$ tongue. The velocity field, whose horizontal component is presented in Figs. 3(b)-3(d) at three different times, is analyzed with the time-frequency function, $S_{\mathbf{u}}(t, \omega)=\left\langle\left|\int_{-\infty}^{+\infty} d t^{\prime} h\left(t-t^{\prime}\right) e^{i \omega t^{\prime}} u\right|^{2}+\right| \int_{-\infty}^{+\infty}$ $\left.\left.d t^{\prime} h\left(t-t^{\prime}\right) e^{i \omega t^{\prime}} w\right|^{2}\right\rangle_{x, z}$, which is plotted in Fig. 3(e). The function $h$ is a Hanning window of $80 T_{0}$. This time window is wide enough to resolve subharmonic frequencies and narrow enough to consider forcing amplitude constant within its duration (increase of $0.85 \mathrm{~mm}$ ). The horizontal dark red line at $\omega / N=0.62$ in Fig. 3(e) corresponds to the forcing frequency. One distinguishes several different regimes: the linear regime [Fig. 3(b)] with a monochromatic response

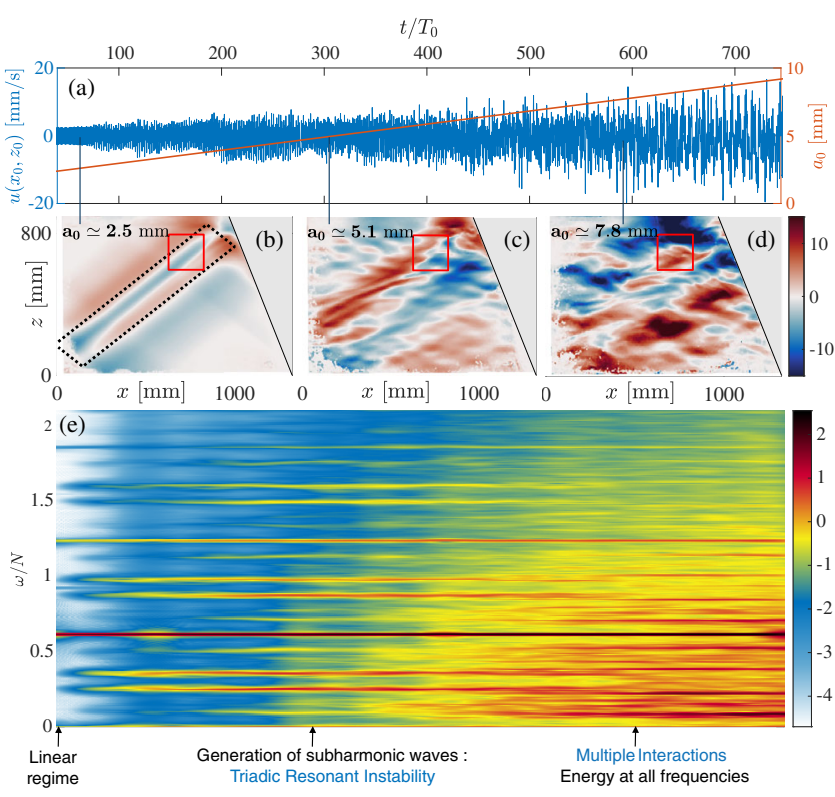

FIG. 3. (a) Amplitude ramp (red) and horizontal velocity component $u(x, z, t)$ measured in a point at the center of the red square shown in (b). (b)-(d) Three snapshots of the horizontal velocity fields (in $\mathrm{mm} / \mathrm{s}$ ) measured when $a=2.5,5.1$, and $7.8 \mathrm{~mm}$. e) Time-frequency diagram $S_{\mathbf{u}}(t, \omega)$ of the velocity field of the red square domain (in logarithmic scale). $\alpha=21.4^{\circ}$, $L=1328 \mathrm{~mm}, N=0.7 \mathrm{rad} / \mathrm{s}$, and $\omega_{0} / N=0.62$.

leading to a well-defined single attractor. Figure 3(c) corresponds to a TRI-perturbed IWA: the two subharmonic waves (around $\omega_{-} / N=0.25$ and $\omega_{+} / N=0.37$ ) apparent at this time in Fig. 3(e) clearly satisfy the temporal resonance condition with $\omega_{0}$. We also observe $\omega_{0}+\omega_{-}$and $\omega_{0}+\omega_{+}$, the signatures of the interaction of these subharmonics with the attractor. The third and turbulentlike regime [Fig. 3(d)] is characterized by a broadband Fourier spectrum and a spatial pattern in which no single IWA is evident. When the amplitude is further increased, the enrichment of the spectrum is progressive, contrary to numerical simulations of the elastic plates [30]. Although some peaks are still visible at large forcing (especially at the forcing frequency), a continuous spectrum is nonetheless established. We estimate (see Supplemental Material [22]) that the nonlinear interaction between waves typically occurs at a timescale 1-10 times larger than the linear one, thus leading to a strong wave turbulent regime.

Spatial spectra.-Figure 4(a) presents the evolution of the wave number power spectrum $E_{k}(k)=k\left[\left\langle|\hat{u}(k, \theta, t)|^{2}+\right.\right.$ $\left.\left.|\hat{w}(k, \theta, t)|^{2}\right\rangle_{\theta, t}\right] / \mathcal{A}$ as the forcing amplitude is gradually increased. Here, $k=|\mathbf{k}|$ is the wave number, $\mathcal{A}$ is the area of the trapezoidal domain, and $\hat{u}$ and $\hat{w}$ are the Fourier transforms of the components of the velocity field. To improve the signal-to-noise ratio, the spectrum has been averaged over all angles $\theta$ and over many forcing periods, as indicated by $\langle\cdot\rangle_{\theta, t}$. The analysis has been performed only on the low frequency band (i.e., $\omega<N$ ), where propagative waves are predominant (see Ref. [31] and Fig. 7 in the Supplemental Material [22]). 
In the linear regime, IWAs have been shown to have an exponential power spectrum $E_{0} \exp (-\beta k)$, when dissipation due to the lateral walls dominates that of the bulk [24]. This behavior is indeed observed at small amplitude $(a=2 \mathrm{~mm})$, as shown by the thick dash-dotted blue line. When $a$ is slightly increased, the attractor is destabilized by TRI and a small peak is visible around $k=150 \mathrm{rad} / \mathrm{m}$, corresponding to a $4 \mathrm{~cm}$ wavelength. Such subharmonic waves have a wavelength smaller than the typical width of the attractor [4]. Further increasing $a$, the peak not only moves toward smaller values of $k$ but also widens.

This first observation can be explained as the coupling between the spectral energy transfer due to the linear focusing and the one due to nonlinear interactions. The energy focusing is now balanced both by dissipation and by extraction via TRI leading to a wider beam within the IWA. The primary wave, having a larger wavelength, generates secondary waves with larger wavelengths as well (see Supplemental Material [22]). Moreover, because their group velocity $c_{g}=N \cos \theta / k$ is larger, subharmonic waves rapidly fill the whole tank.

At large forcing, these different ingredients lead to a richer spectrum that is compatible with a power law decay $E_{k} \sim k^{-3}$, as shown in Fig. 4(a). The same exponent has been obtained in various numerical simulations with different forcing mechanisms [32,33].

We have gone on to examine these analyses for experiments with different forcing and geometrical parameters. We have studied a strongly focused IWA that corresponds to a larger Lyapunov exponent than for the weakly focused IWA shown in Fig. 4(a). Taking advantage of the band structure revealed in the kicked attractor experiment, we also forced the stratified fluid via a white noise filtered in the frequency range of the $(1,1)$ attractor shown in Fig. 2(h). In both cases, the exponent -3 is robust, as shown in Fig. 4(b).

Conclusion.-We have performed the first experimental measurements of internal wave turbulence. The trapezoidal, stably stratified domain is a robust experimental setup to study nonlinearly interacting internal waves. Different forcings, whether monochromatic or frequency modulated, lead to a power spectrum with a well-defined power law.

Future work will focus on examination of the frequency spectra. For frequencies below the buoyancy frequency $N$, one expects a $\omega^{-2}$ spectrum similar to the Garrett and Munk spectrum of observed oceanic internal waves [34]. This is often used as a representative statistical description of the internal wave field in studies of nonlinear interaction, despite only an approximate description. For frequencies above $N$, a steeper $\omega^{-4}$ spectrum has been recently reported in numerical simulations [35]. Whether or not both spectra are manifest in experiments remains an open question. To be closer to oceanic circumstances, experiments are being designed with a less constrained geometrical setup that would allow for three-dimensional dynamics.
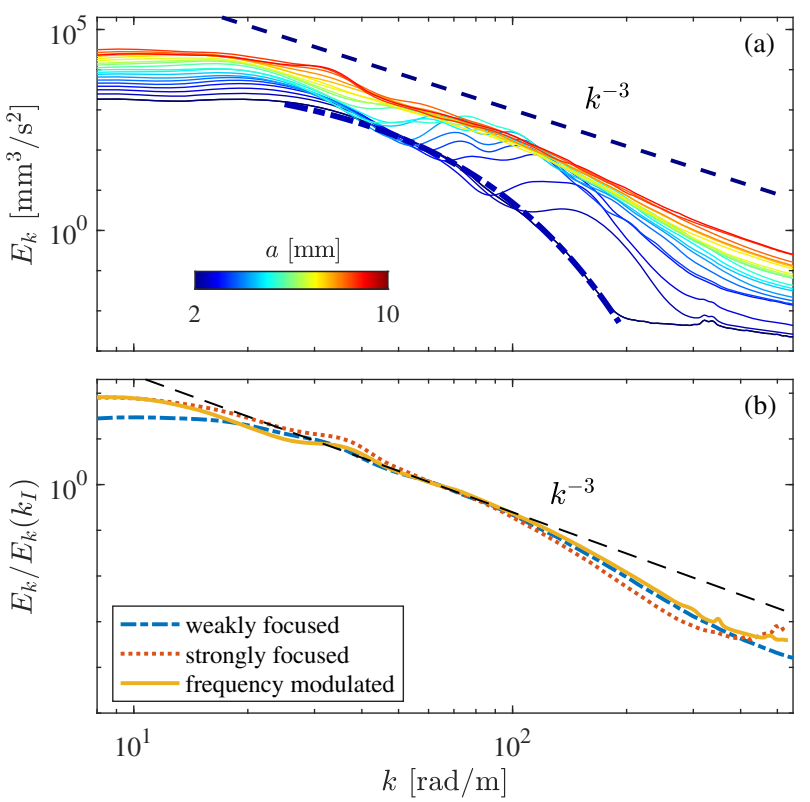

FIG. 4. (a) Power spectral density $E_{k}(k)=k\left[\left\langle|\hat{u}(k, \theta, t)|^{2}+\right.\right.$ $\left.\left.|\hat{w}(k, \theta, t)|^{2}\right\rangle_{\theta, t}\right] / \mathcal{A}$ as a function of the wave number $k$ for the experiment shown in Fig. 3. The spatial Fourier transform of the components of the velocity field $\hat{u}$ and $\hat{w}$ are computed using a Hanning window. The different curves correspond to an increase of the amplitude $a$ from 2 to $10 \mathrm{~mm}$ (that lasts for 750 periods), with an average over 35 forcing periods for each curve. The thick dash-dotted blue line corresponds to a fit, whose exponential shape is predicted for a linear IWA. (b) Stationary spectrum averaged over 100 periods for different forcings. The different experimental parameters are given in the Supplemental Material [22]. In both panels, the dashed line shows the power law $E_{k} \simeq k^{-3} . k_{I} \simeq 60 \mathrm{rad} / \mathrm{m}$ is taken in the center of the inertial range.

We are indebted to C. Brouzet, E. Ermanyuk, C. Herbert, L. Maas, L. Saint Raymond, and B. Sutherland for useful discussions. This work was supported by the Grant No. ANR-17-CE30-0003 (DisET) and by the LABEX iMUST (ANR-10-LABX-0064) of Université de Lyon, within the program Investissements d'Avenir (ANR-11IDEX-0007), operated by the French National Research Agency (ANR). This work was supported by a grant from the Simons Foundation (651475, TD). It has been achieved thanks to the resources of PSMN from ENS de Lyon.

[1] B. R. Sutherland, Internal Gravity Waves (Cambridge University Press, Cambridge, 2010).

[2] M. H. Alford et al., Nature (London) 521, 65 (2015).

[3] M. J. Mercier, L. Gostiaux, K. Helfrich, J. Sommeria, S. Viboud, H. Didelle, S. J. Ghaemsaidi, T. Dauxois, and T. Peacock, Geophys. Res. Lett. 40, 5704 (2013).

[4] T. Dauxois, S. Joubaud, P. Odier, and A. Venaille, Annu. Rev. Fluid Mech. 50, 131 (2018).

[5] H. van Haren, J. Plankton Res. 36, 1124 (2014). 
[6] E. C. Reid, T. M. DeCarlo, A. L. Cohen, G. T. F. Wong, S. J. Lentz, A. Safaie, A. Hall, and K. A. Davis, Limnol. Oceanogr. 64, 1949 (2019).

[7] A. Campagne, B. Gallet, F. Moisy, and P.-P. Cortet, Phys. Rev. E 91, 043016 (2015).

[8] G. D. Portwood, S. M. de Bruyn Kops, and C. P. Caulfield, Phys. Rev. Lett. 122, 194504 (2019).

[9] S. Nazarenko, Wave Turbulence, Lecture Notes Physics Vol. 825 (Springer, Berlin; Heidelberg, 2011).

[10] L. Deike, C. Laroche, and E. Falcon, Europhys. Lett. 96, 34004 (2011).

[11] M. Berhanu, E. Falcon, G. Michel, C. Gissinger, and S. Fauve, Europhys. Lett. 128, 34001 (2020).

[12] E. Yarom and E. Sharon, Nat. Phys. 10, 510 (2014).

[13] E. Yarom, A. Salhov, and E. Sharon, Phys. Rev. Fluids 2, 122601(R) (2017).

[14] E. A. Kuznetsov, J. Exp. Theor. Phys. 93, 1052 (2001).

[15] B. Miquel, A. Alexakis, C. Josserand, and N. Mordant, Phys. Rev. Lett. 111, 054302 (2013).

[16] A. Picozzi, J. Garnier, T. Hansson, P. Suret, S. Randoux, G. Millot, and D. N. Christodoulides, Phys. Rep. 542, 1 (2014).

[17] S. Galtier and S. V. Nazarenko, Phys. Rev. Lett. 119, 221101 (2017).

[18] Y. V. Lvov and E. G. Tabak, Phys. Rev. Lett. 87, 168501 (2001).

[19] L. R. M. Maas and F. P. A. Lam, J. Fluid Mech. 300, 1 (1995).

[20] C. Brouzet, E. Ermanyuk, S. Joubaud, G. Pillet, and T. Dauxois, J. Fluid Mech. 811, 544 (2017).

[21] L. R. M. Maas, D. Benielli, J. Sommeria, and F. P. A. Lam, Nature (London) 388, 557 (1997).
[22] See Supplemental Material at http://link.aps.org/ supplemental/10.1103/PhysRevLett.124.204502 for experimental details, movies, pictures, and additional measurements of the internal wave attractor experiment. Notations are the same as in the present paper, which includes Ref. [23].

[23] E. Hopfinger, J.-B. Flor, J.-M. Chomaz, and P. Bonneton, Exp. Fluids 11, 255 (1991).

[24] F. Beckebanze, C. Brouzet, I. N. Sibgatullin, and L. R. M. Maas, J. Fluid Mech. 841, 614 (2018).

[25] G. Davis, T. Dauxois, T. Jamin, and S. Joubaud, J. Fluid Mech. 880, 743 (2019).

[26] G. Pillet, E. V. Ermanyuk, L. Maas, I. Sibgatullin, and T. Dauxois, J. Fluid Mech. 845, 203 (2018).

[27] B. Bourget, T. Dauxois, S. Joubaud, and P. Odier, J. Fluid Mech. 723, 1 (2013).

[28] H. Scolan, E. Ermanyuk, and T. Dauxois, Phys. Rev. Lett. 110, 234501 (2013).

[29] Y. Colin de Verdière and L. Saint Raymond, Comm. Pure Appl. Math. 73, 421 (2020).

[30] C. Touzé, S. Bilbao, and O. Cadot, J. Sound Vib. 331, 412 (2012).

[31] C. Brouzet, E. V. Ermanyuk, S. Joubaud, I. Sibgatullin, and T. Dauxois, Europhys. Lett. 113, 44001 (2016).

[32] T. Le Reun, B. Favier, and M. Le Bars, J. Fluid Mech. 840, 498 (2018).

[33] P. Bouruet-Aubertot, J. Sommeria, and C. Staquet, Dynamics of Atmospheres and Oceans 23, 357 (1996).

[34] C. J. Garrett and W. Munk, J. Geophys. Res. 80, 291 (1975).

[35] P. C. di Leoni and P. D. Mininni, Phys. Rev. E 91, 033015 (2015). 